\title{
Polypoidal Choroidal Vasculopathy - A Type I Polypoidal Subretinal Neovasculopathy
}

\author{
Gregg T. Kokame*
}

\begin{abstract}
Division of Ophthalmology, Department of Surgery, University of Hawaii John A Burns School of Medicine, Honolulu, Hawaii; Retina Consultants of Hawaii, Honolulu, Hawaii, The Retina Center at Pali Momi, An Affiliation of Hawai'i Pacific Health, USA
\end{abstract}

\begin{abstract}
This 47 year old female developed the new onset of a polypoidal subretinal neovascular membrane in the left eye 13 years after having polypoidal choroidal vasculopathy (PCV) treated in the right eye. The indocyanine green (ICG) angiography of the left eye at initial presentation showed a normal choroidal vascular pattern without PCV. The new development of a PCV complex on ICG angiography demonstrates that PCV is truly a type of subretinal neovascularization, and not a choroidal vascular abnormality. The optical coherence tomography shows that the polypoidal vascular complex lies above Bruch's membrane and beneath the retinal pigment epithelium, and not within the choroid. Treatment with high dose ranibizumab $(2.0 \mathrm{mg})$ resulted in excellent polyp closure and regression of the branching vascular network. The documented new development of polypoidal subretinal vessels on ICG angiography and the response to ranibizumab supports that PCV is a polypoidal neovasculopathy (PNV).
\end{abstract}

Keywords: Subretinal neovascularization, polypoidal choroidal vasculopathy, ranibizumab, serous retinal detachment, branching vascular network, macular edema.

\section{INTRODUCTION}

Polypoidal choroidal vasculopathy (PCV) was initially described by Yannuzzi as a cause of exudation and bleeding in the macula [1-2]. Most reports previously have been uncertain as to whether PCV represents a choroidal vascular abnormality or a type of subretinal neovascularization [3]. Recently, Freund [4] has proposed that PCV is a form of type I subretinal neovascularization according to the classification system of Gass [5]. This case report shows that PCV is truly a type of subretinal neovascularization, as it developed in an eye with a previously normal indocyanine green (ICG) angiogram 13 years previously. The response to high dose ranibizumab therapy further supports that PCV is not a pre-existing choroidal vascular abnormality, but a type of neovascularization responsive to antiangiogenesis. The optical coherence tomography localizes the PCV to be a neovascular membrane above Bruch's membrane and beneath the retinal pigment epithelium (RPE), and not within the choroid.

\section{CASE REPORT}

A 47 year old Filipino female presented with the sudden onset of decrease in vision in the right eye in 1998. Visual acuity was: OD 20/70 OS 20/20. Fundus exam showed large orange subretinal vascular structures in the extrafoveal region with serous detachment in the fovea (Fig. 1). ICG angiography showed a temporal branching vascular network

*Address correspondence to this author at the Retina Center at Pali Momi, 98-1079 Moanalua Road Suite 470, Aiea, Hawaii 96701, USA; Tel: 1-808487-3699; Fax: 1-808-487-8928; E-mail: retinahi@aol.com with extrafoveal polyps (Fig. 2A). This eye underwent macular laser and has maintained 20/20 vision after 14 years. The ICG angiogram of the left eye showed a completely normal choroidal vasculature without a PCV complex (Fig. 2B).

Thirteen years later in 2011 the patient noted the sudden onset of a black and grey spot in the left eye for 2 weeks. ICG angiography confirmed the presence of a new peripapillary polypoidal vascular complex (Fig. 3A), that was not present on ICG angiography 13 years earlier. Optical coherence tomography corresponding to the polyps showed that the subretinal neovascular complex lies above Bruch's membrane and below the retinal pigment epithelium (Fig. 3B). This patient was treated with intravitreal $2.0 \mathrm{mg}$ ranibizumab in the PEARL2 study with remarkable resolution of the large vascular polyps, the retinal pigment epithelial detachment, the serous retinal detachment, and the branching vascular network (BVN) (Fig. 3C).

\section{DISCUSSION}

Polypoidal choroidal vasculopathy (PCV) is a term originated by Yannuzzi in his initial description of leaking and bleeding complications in the macula associated with a polypoidal subretinal vascular complex [1]. It has been uncertain as to whether this represents a choroidal vascular abnormality, a degenerative vascular lesion, or a type of subretinal neovascularization [3]. This case supports that PCV is a type of subretinal neovascularization, as the vascular complex was not present on ICG angiography 13 years prior to developing symptoms in the left eye (Figs. 2B, 3A). Freund [4] has emphasized that this vascular complex is 


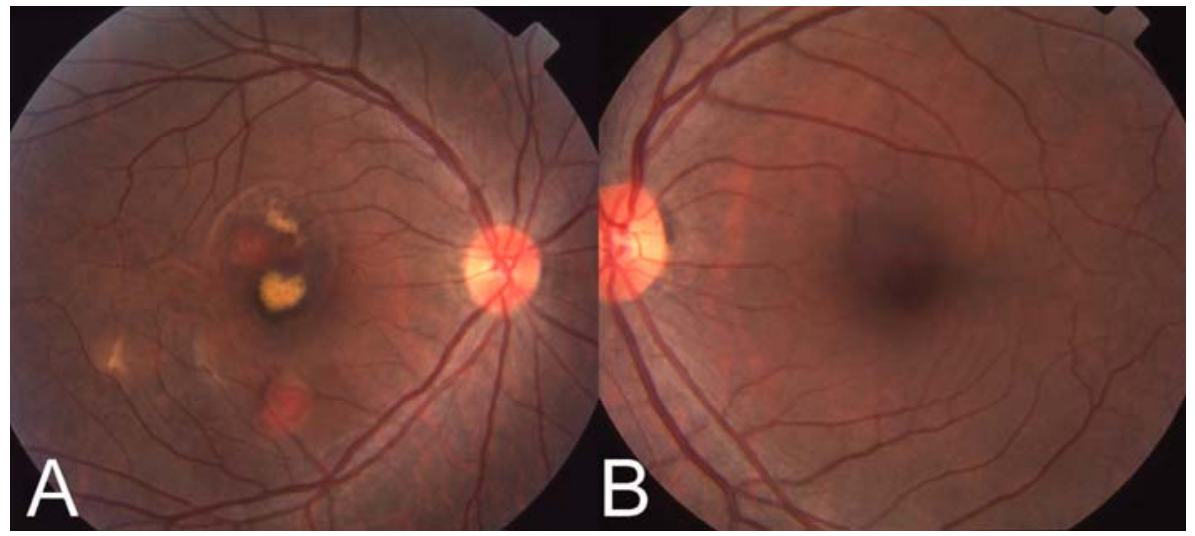

Fig. (1). (A) Color fundus photograph of right eye. Note the large orange subretinal vascular lesions superotemporal and inferior to the center of the fovea, and serous detachment involving the central fovea. (B) Color fundus photograph of the left eye. Note the lack of any polypoidal vascular abnormality.

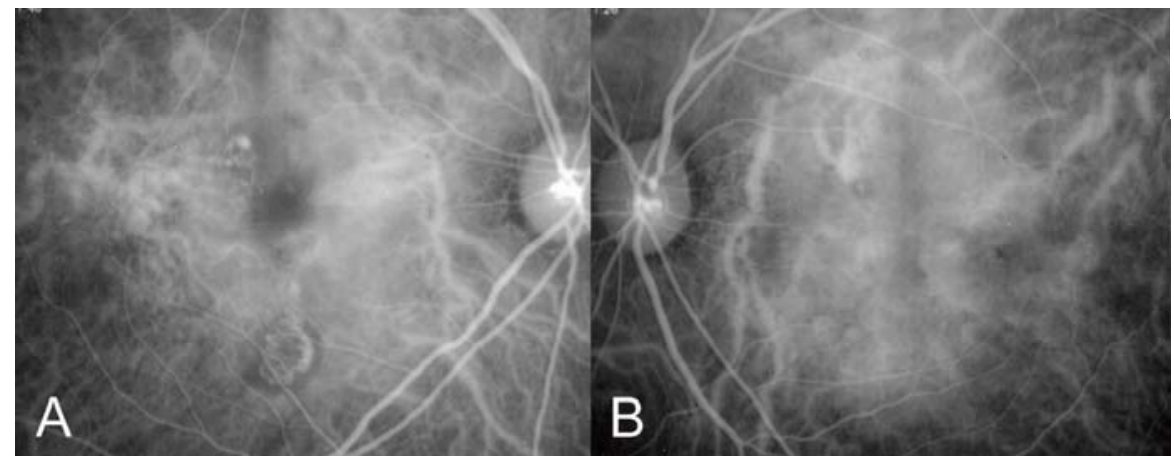

Fig. (2). (A) ICG angiogram of the right eye. Note the temporal branching vascular network and the extrafoveal superotemporal and inferior polyps. (B) ICG angiogram of the left eye. Noted the normal choroidal vasculature and lack of any polypoidal complex.

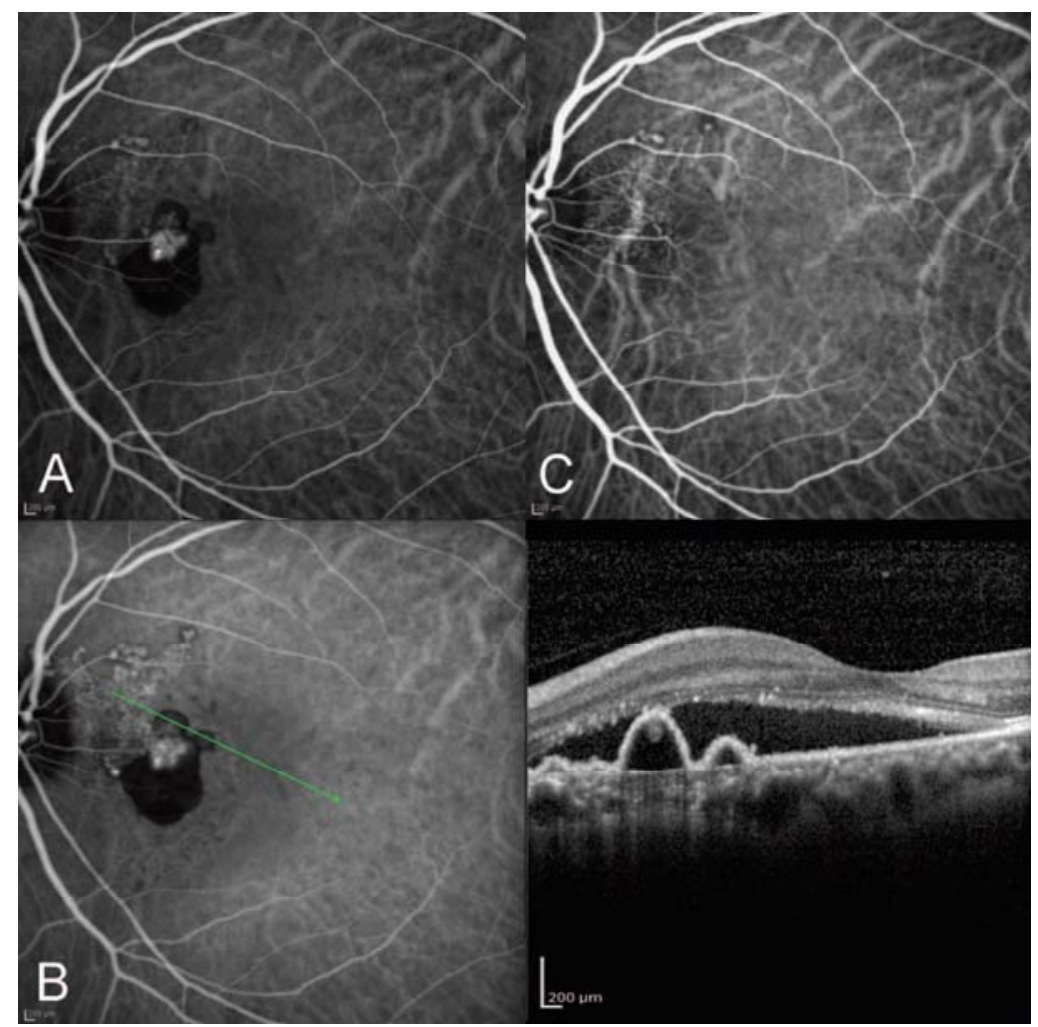

Fig. (3). (A) ICG angiogram of the left eye. Note the new onset of polypoidal subretinal neovascularization. (B) OCT of the left eye corresponding to the polypoidal complex. Note the subretinal neovascularization lying above Bruch's membrane and below the retinal pigment epithelium. (C) ICG angiography after high dose ranibizumab showing resolution of the polyps and RPED with decrease in the branching vascular network. 
a variant of type I subretinal neovascularization as described by the classification system of Gass [5]. The vascular complex, including the branching vascular network and the polyps, lies above Bruch's membrane and beneath the retinal pigment epithelium. This was also confirmed by the optical coherence tomography findings associated with the ICG angiogram in this case (Fig. 3B). The disappearance of the vascular complex on high dose antiangiogenic therapy (2.0 mg ranibizumab) also supports that polypoidal vascular lesions are a type of subretinal neovascularization. These vessels may be better described as a polypoidal neovasculopathy (PNV), as proposed by Freund [4], rather than polypoidal choroidal vasculopathy, as the vessels are neovascular and lie above Bruch's membrane and beneath the retinal pigment epithelium, and not within the choroid.

This location of the polypoidal vascular complex beneath the retinal pigment epithelium may have important implications for treatment. This patient was treated as part of a clinical trial of high dose ranibizumab for exudative and hemorrhagic complications of PCV. Although ranibizumab at the usual $0.5 \mathrm{mg}$ dose of ranibizumab does result in decreased leakage, macular edema and hemorrhage, as shown in the PEARL trial [6], the PCV complex decreases in only $33 \%$ of cases after 6 monthly injections. In this case treated with high dose ranibizumab $(2.0 \mathrm{mg})$, there was a marked anatomic response of the PCV complex, in addition to the decrease in exudation and leakage. Although this is a small study of high dose treatment, drugs either at a higher dose or with a better ability to penetrate the retinal pigment epithelium, could potentially be more effective at anatomic closure and treatment of PCV.

\section{CONFLICT OF INTEREST}

The author confirms that this article content has no conflict of interest.

\section{ACKNOWLEDGEMENTS}

Declared none.

\section{REFERENCES}

[1] Yannuzzi LA, Sorensen J, Spaide RF, Lipson B. Idiopathic polypoidal choroidal vasculopathy. Retina 1990; 10: 1-8.

[2] Yannuzzi LA, Ciardella A, Spaide RF, et al. The expanding clinical spectrum of idiopathic polypoidal choroidal vasculopathy. Arch Ophthalmol 1997; 115: 478-5.

[3] Laude A, Cackett PD, Vithana E, et al. Polypoidal choroida vasculopathy and neovascular age-related macular degeneration: Same or different disease? Prog Retin Eye Res 2010; 29(1): 19-29.

[4] Freund KB, Zweifel SA, Engelbert M. Editorial. Do we need a new classification for choroidal neovascularization in age-related macular degeneration? Retina 2010; 30: 1333-49.

[5] Gass JD. Stereoscopic atlas of macular diseases. In: $4^{\text {th }}$ Ed., St Louis, Missouri, C. V. Mosby 1997; pp. 26-30.

[6] Kokame GT, Yeung L, Lai JC. Continuous anti-VEGF treatment with ranibizumab for polypoidal choroidal vasculopathy: 6 month results. Br J Ophthalmol 2010; 94(3): 297-301. 